\title{
RESEARCH
}

Open Access

\section{Efficacy of topical and systemic transplantation of mesenchymal stem cells in a rat model of diabetic ischemic wounds}

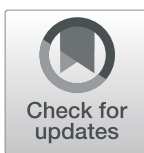

Jianxin Yan ${ }^{1,2,3+}$, Jiaji Liang ${ }^{1,2,3+}$, Yingxuan Cao ${ }^{1,2,3}$, Mariya M. El Akkawi ${ }^{1,2,3}$, Xuan Liao ${ }^{1,2,3}$, Xiaojia Chen ${ }^{4,5,6,7}$, Chengzhi Li $i^{8}$, Kecheng Li ${ }^{1,2,3}$, Guanghui Xie ${ }^{1,2,3}$ and Hongwei Liu ${ }^{1,2,3^{*}}$

\begin{abstract}
Background: Mesenchymal stem cells (MSCs) exert positive effects in chronic wounds. However, critical parameters, such as the most effective administration routes, remain unclear. Accordingly, the purpose of this study was to compare the effects of topical and systemic transplantation MSCs on diabetic ischemic wound healing and explored the underlying mechanisms.
\end{abstract}

Method: A diabetic ischemic wound model was created on the dorsal foot of type 2 diabetes mellitus (T2DM) rat. Bone marrow-derived mesenchymal stem cells (BM-MSCs) were administered via two routes: topical injection and intravenous (IV) infusion. Wound healing outcomes and blood glucose level were assessed dynamically. Meanwhile, blood flow recovery was evaluated in ischemic gastrocnemius muscles. The homing and transdifferentiation of mKate2-labeled BM-MSCs were assessed by fluorescence imaging and immunohistochemistry (IHC) analysis.

Result: Both topical and systemic treatments had a positive effect on the diabetic ischemic wound showing a significant reduction in wound area at day 14 . Histological results showed an increase in the length of epithelial edges, collagen content, microvessel density in the wound bed, and a higher expression of vascular endothelial growth factor (VEGF). Meanwhile, systemic administration can ameliorate hyperglycemia and improve the blood perfusion of the ischemic hindlimb. BM-MSCs administered systemically were found distributed in wounded tissue and transdifferentiated into endothelial cells. Furthermore, BM-MSCs stimulated angiogenesis at wound sites by downregulating phosphatase and tensin homolog (PTEN) and activation of AKT signaling pathway.

Conclusions: The results demonstrated that both transplantation delivery method (topical and systemic) of BMMSCs accelerated wound healing remarkably under pathological conditions. Nevertheless, systemic administration has the potential to ameliorate hyperglycemia and repair the damaged tissue.

Keywords: Bone marrow-derived mesenchymal stem cells, Type 2 diabetes mellitus, Critical limb ischemia, Diabetic foot ulcers, Chronic wounds

\footnotetext{
* Correspondence: liuhongwei0521@hotmail.com

†Jianxin Yan and Jiaji Liang contributed equally to this work.

'Department of Plastic Surgery, The First Affiliated Hospital of Jinan

University, Guangzhou 510630, People's Republic of China

${ }^{2}$ Innovative Technology Research Institute of Plastic Surgery, Guangzhou

510630, People's Republic of China

Full list of author information is available at the end of the article
}

\section{$\triangle B M C$}

(c) The Author(s). 2021 Open Access This article is licensed under a Creative Commons Attribution 4.0 International License, which permits use, sharing, adaptation, distribution and reproduction in any medium or format, as long as you give appropriate credit to the original author(s) and the source, provide a link to the Creative Commons licence, and indicate if changes were made. The images or other third party material in this article are included in the article's Creative Commons licence, unless indicated otherwise in a credit line to the material. If material is not included in the article's Creative Commons licence and your intended use is not permitted by statutory regulation or exceeds the permitted use, you will need to obtain permission directly from the copyright holder. To view a copy of this licence, visit http://creativecommons.org/licenses/by/4.0/ The Creative Commons Public Domain Dedication waiver (http://creativecommons.org/publicdomain/zero/1.0/) applies to the data made available in this article, unless otherwise stated in a credit line to the data. 


\section{Introduction}

Type 2 diabetes mellitus (T2DM) is the most prevalent form of diabetes, accounting for 90 to $95 \%$ of existing cases. T2DM is characterized by insulin resistance in the target tissue, a relative lack of insulin secretion, and subsequent decline in the $\beta$ cell function in the pancreas [1]. In clinical practice, patients suffering from T2DM frequently present with critical limb ischemia and foot ulcer [2]. Diabetic foot ulcers (DFUs) are one of the most common types of chronic wounds with pathological hallmarks of decreased vascularization, elevated oxidative stress, and infection [3]. The occurrence of DFUs is closely related to peripheral arterial disease (PAD), which is a process of chronic atherosclerosis, mainly resulting in the narrowing of the lower limbs' peripheral arterial vascular system. Clinical data suggested that DFUs is one of the major causes of non-traumatic lower limb amputation, mainly due to microvascular and macrovascular complications [4]. At present, the management of DFUs includes glycemic control, pharmacological therapy, improving vascularization, debridement, "offloading" strategies, wound dressings, negative pressure wound therapy, maggot therapy, growth factors, and skin substitutes [5]. However, these conventional treatments have certain limitations.

In recent years, studies showed the effectiveness of mesenchymal stem cells (MSCs) in treating DFUs [6, 7]. As one of multipotent adult stem cells, MSCs can differentiate into the different cell lineages in damaged tissues by activating endogenous progenitor cells and secreting various factors and regulating their local environment $[8,9]$. Therefore, MSCs have great application prospects in the regenerative treatment of damaged tissues and chronic wounds, such as critical limb ischemia [10], diabetic foot [11], and pressure ulcers [12]. Although a spectacular number of MSCs studies have already been conducted, the safety and efficacy of MSCs still remains uncertain: in terms of source, management prior to administration, nature (autologous or allogenic), optimum dosage, and route and timing of administration. Systemic administration and topical delivery are both important methods for MSC transplantation. Intravenous (IV) infusion is the common method of clinical systemic administration due to being a relatively easy and least invasive procedure [13]. However, it was reported that MSCs follow a process known as "lung entrapment" which leads to cells mainly distributed in the lungs and rarely homing to the target tissue after IV infusion [14, 15]. Lung entrapment is also considered to be a first-pass effect in the early stages $(1 \mathrm{~h})$ after IV infusion and MSCs homing in other tissues and organs over time [16]. Whether MSCs can reach target tissues through IV infusion remains controversial. Hence, recent research on MSCs therapy of wounds focused on topical transplantation, which directly injects MSCs into the target tissue. Regardless, both systemically and locally administered MSCs promote wound healing under physiological conditions, but their mechanism may be different [17]. As a result, the optimal route of transplantation of MSCs for chronic wounds requires further research.

In this study, a diabetic ischemic wound model on rat dorsal foot was designed to mimic the pathophysiology of clinical patients who suffer from DFUs. Bone marrowderived MSCs (BM-MSCs) are currently the most frequently used MSCs source, so we transplanted BM-MSCs topically and systemically to investigate the proper administration method for chronic wound therapy. The potential therapeutic mechanisms were further investigated.

\section{Materials and methods} Isolation, culture, identification, and labeling of BM-MSCs BM-MSCs were collected by flushing the femurs and tibias of 4-week-old male Sprague-Dawley rat (Guangdong Medical Laboratory Animal Center). These cells were cultured at $37{ }^{\circ} \mathrm{C}$ with $5 \% \mathrm{CO}_{2}$ in a basic medium, consisting of high glucose Dulbecco's modified Eagle's medium (DMEM; Product \# 10-013-CVR; Corning), 10\% fetal bovine serum (FBS; Product \# 04-001-1ACS; Biological Industries), and $1 \%$ penicillin-streptomycin (pen/strep; Product \# SV30010; HyClone). Nonadherent cells were removed after $48 \mathrm{~h}$ of incubation and a fresh medium was added. The medium was changed every 48 or $72 \mathrm{~h}$ and further propagated the adherent spindle-shaped cells for three passages.

BM-MSCs were identified by immune phenotypic analyses and differentiation studies as described previously [18]. We phenotypically characterized the cultured cells for the expression of MSC markers by flow cytometry as $\mathrm{CD} 29^{+} \mathrm{CD} 44^{+} \mathrm{CD} 90^{+} \mathrm{CD} 34^{-} \mathrm{CD} 45^{-}$. On the other hand, differentiation into adipocytes, osteocytes, and chondrocytes determined their functional characterization. Three days after transfecting mKate2-expressing-lentivirus to BMMSCs (passage 2) at 70\% cell confluence, fluorescence microscope (IX51; Olympus) was used for the detection of infection efficiency, and the cells were cultured successively.

\section{Diabetic ischemic wound model}

Seven-week-old $200 \mathrm{~g}$ male Sprague-Dawley rats (Guangdong Medical Laboratory Animal Center) were used for all studies, maintained on a 12-h:12-h light-dark cycle under ad libitum feeding and water consumption. The animal experiment protocol was approved by the Laboratory Animal Ethics Committee of Jinan University. The T2DM model was induced in rats with fat-fed (45\% fat/35\% carbohydrate $/ 20 \%$ protein; and $4.73 \mathrm{kcal} / \mathrm{g}$ ) 2 weeks and then streptozotocin injected intraperitoneally twice $(24 \mathrm{~h}$ apart) at a dose of $35 \mathrm{mg} / \mathrm{kg}[19,20]$. Intraperitoneal glucose tolerance tests (IPGTTs), intraperitoneal insulin 
tolerance tests (IPITTs), and serum insulin were performed 1 week after STZ injection to confirm the T2DM rat model. Blood glucose monitor (Roche Diagnostics $\mathrm{GmbH}$, Mannheim, Germany) was used to measure blood glucose. Quantitative measurements of serum insulin were performed by ELISA (Rat Insulin Elisa Kit; Wuxi Donglin Sci \& Tech Development Co., Ltd., Jiangsu, China).

Twelve weeks following the success of T2DM model, serum nitric oxide (NO) (Nitric Oxide assay kit; NanJing JianCheng Bioengineering Institute Co., Ltd., Nanjing, China), serum endothelin-1 (ET-1) (Rat Endothelin-1 ELISA Kit; Wuhan Huamei Bioengineering Co., Ltd., Wuhan, China), and oil red $\mathrm{O}$ staining (Wuhan servicebio technology Co., Ltd., Wuhan, China) were performed to confirm the vasculopathy. Meanwhile, body weight, 24-h water, and feed intake were compared between normal and T2DM rats. After that, a single round fullthickness skin wound was created using disposable 5 mm skin biopsy punch in T2DM rats after the ligation of the right femoral artery to created a diabetic ischemic wound model on the dorsal hindfoot.

\section{Transplantation and tracing of BM-MSCs}

Rats with diabetic ischemic wounds were randomly divided into a PBS control group, a BM-MSC topically treated group, and a BM-MSC IV-treated group. Topically treated rats received $1 \times 10^{6} \mathrm{BM}-\mathrm{MSCs}$ in $100 \mu \mathrm{L}$ of PBS intradermal injections at four injection sites around the wound immediately after creation [21]. In contrast, IV treated rats received $5 \times 10^{6} \mathrm{BM}-\mathrm{MSCs}$ in $1000 \mu \mathrm{L}$ PBS via the left femoral vein right immediately after wound creation [6]. To evaluate the distribution of MSCs in this model at days 3,7 , and 14, the mKate2-labeled BM-MSCs were applied in the same dosage and administration method as previously described to trace using fluorescence imaging and immunohistochemistry (IHC) analysis.

Rats were perfused with $60 \mathrm{ml}$ of normal saline through the left ventricle followed by $100 \mathrm{ml}$ of $4 \%$ paraformaldehyde. After perfusion, the major organs (Fig. 6a) were dissected and subjected to fluorescence imaging. Fluorescence imaging was performed using In Vivo Xtreme Imaging System (Bruker Corporation, Karlsruhe, Germany) with excitation wavelength $588 \mathrm{~nm}$ and emission wavelength $635 \mathrm{~nm}$. For IHC analysis, the lung, pancreas, and wound sites were detected with antibodies specific against mKate2 (1:4000; Product \# R10367; Invitrogen), the latter subsequently visualized with Alexa Fluor ${ }^{\circ} 647$ Goat Anti-Rabbit IgG antibody (1:500; Product \# A-21245; Invitrogen).

\section{Measurement of wound contraction rate and blood glucose level}

Digital photographs of wounds were taken and blood glucose level was determined at days $0,3,7,10$, and 14 .
The wound area was measured using Image analysis software (National Institutes of Health, Bethesda, MD) by tracing the wound margin. The wound area healing rate was calculated as follows:

Wound area healing rate $(\%)=([$ area of original wound - area of actual wound]/[area of original wound] $) \times 100$.

\section{Color-coded quantitative digital subtraction angiography (q-DSA)}

At days 0,7 , and 14 after injury, double hindlimb q-DSA was performed by abdominal aorta injection of $4 \mathrm{~mL}$ Iohexol $(320 \mathrm{mgI} / \mathrm{ml})$ at $1 \mathrm{~mL} / \mathrm{s}$ speed, $111 \mathrm{kPa}$ pressure. DSA scans were acquired at a frame rate of 6 frames per second (F/s) with the Siemens Artis Zeego C-arm, combined with Artis Zeego software from Siemens Healthineers (version VD11D, Siemens Healthcare, Erlangen, Germany). Image analysis took place using syngo iFlow postprocessing software (Siemens Healthineers, Forchheim, Germany). The gastrocnemius regions of the hindlimb on both sides are selected symmetrically as the region of interest (ROI), and the right ROI peak (PeakROI-R) and left ROI peak (PeakROI-L) are obtained. Then, the ratio of the PeakROI-R to the PeakROI-L was calculated $(\mathrm{rPeak}=$ PeakROI-R/PeakROI-L).

\section{Histological assessment}

The wound (including $2 \mathrm{~mm}$ of the surrounding skin) and the right gastrocnemius tissue samples were harvested at days $3,7,10$, and 14 . The sections of the wound tissue were stained with hematoxylin and eosin (H\&E) and with Masson's trichrome following the manufacturers' protocol (Wuhan servicebio technology Co., Ltd., Wuhan, China) to detect the reepithelialization/granulation tissue formation and collagen deposition, respectively. Meanwhile, the anti-CD31 antibody (1:300; Product \# ab182981; ABcam) and the anti-VEGF antibody (1:200; Product \# ABS82; Sigma-Aldrich) IHC were performed. Goat anti-rabbit IgG H\&L (HRP) (1:200; Product \# ab205718; ABcam) was used as a secondary antibody. The length of both the epithelial edges and the epithelial gap, as well as the area of the granulation tissue on the wound, were determined (Fig. 4a) according to the criteria described previously [22]. Microvessel density is expressed as the number of stained vessels per high power field (HPF) (Fig. 5) refer to previous study [23]. The positive areas of collagen, mKate2, and VEGF staining were calculated using ImageJ analysis software as follows:

Collagen content/area of mKate2 staining/VEGF content $(\%)=([$ area of positive staining $] /[$ area of the entire field of view] $) \times 100$.

\section{Double-labeling IHC for wounded tissue}

After administration on day 14, wound and right gastrocnemius sections were incubated with anti-mKate2 rabbit 
polyclonal antibody (1:4000; Product \# R10367; Invitrogen) and anti-CD31 mouse monoclonal antibody (1:1500; Product \# ab24590; ABcam) overnight at $+4{ }^{\circ} \mathrm{C}$, followed by a further incubation at room temperature for $1 \mathrm{~h}$ with Alexa Fluor 647 goat anti-rabbit IgG antibody (1:500; Product \# A-21245; Invitrogen) and Alexa Fluor 488 goat anti-mouse IgG antibody (1:500; Product \# ab150117; ABcam). Nuclei were counterstained with DAPI stain (Product \# G1012; Servicebio).

\section{Western blot analysis}

Total protein was extracted from samples of wound by Total Protein Extraction Kit (Beyotime Institute of Biotechnology, Shanghai, China) at day 14 posttreatment.
Equal amounts of total protein were separated on $10 \%$ SDS-PAGE and transferred to nitrocellulose membranes. Membranes were incubated overnight at $4{ }^{\circ} \mathrm{C}$ with monoclonal antibody against VEGF (Product \# ABS82), phosphorylation of AKT (p-AKT) (Product \# SAB4504331), AKT (Product \# SAB4500797), phosphatase and tensin homolog (PTEN) (Product \# SAB4300337), and $\beta$-actin (Product \# ABT264) (all 1: 1000; Sigma-Aldrich). Then, the membranes were incubated with HRP-conjugated anti-rabbit (1:5000; Product \# ab205718; ABcam).

\section{Statistical analysis}

All data were presented as means \pm SD. Two analytic methods, $t$ test and ANOVA, were used to analyze the
A

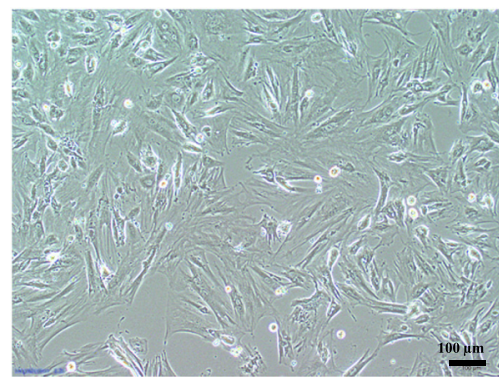

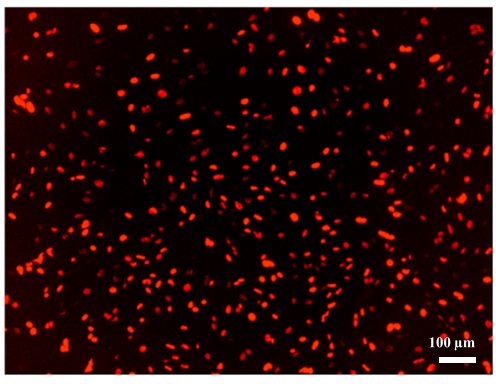

C

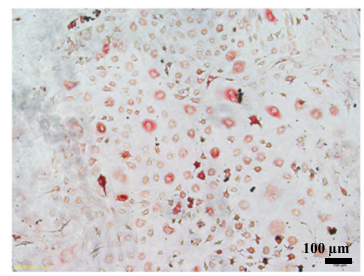

ADIPOGENESIS

D

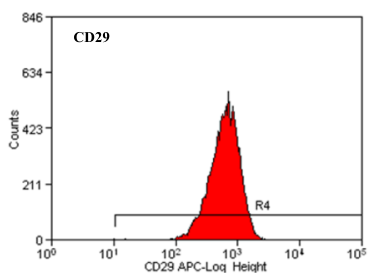

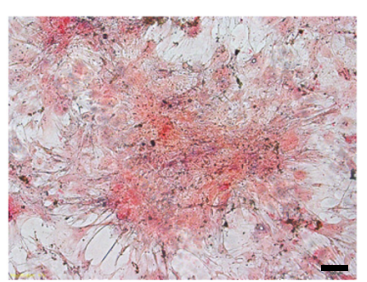

OSTEOGENESIS

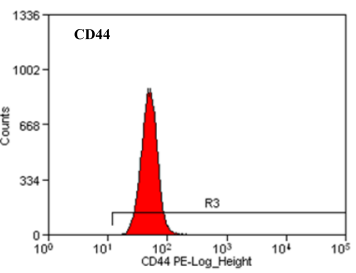

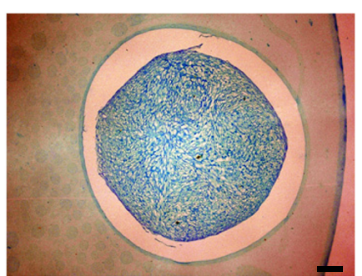

CHONDROGENESIS

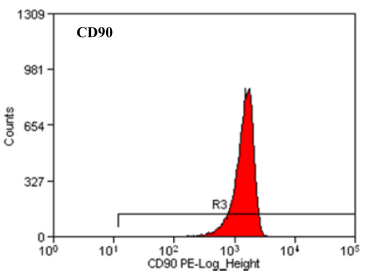

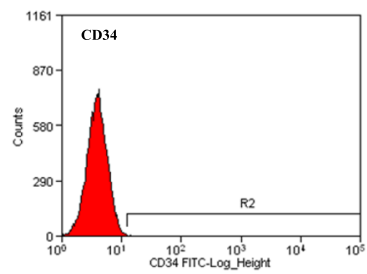

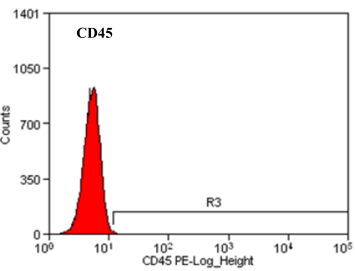

Fig. 1 Characterization of rat BM-MSCs. a Brightfield image of BM-MSCs in culture. $\mathbf{b}$ A fluorescent image of transduced BM-MSCs expressing mKate2 (red fluorescence). c Adipogenic differentiation was revealed with oil red O staining. Osteogenic differentiation was confirmed by alizarin red staining. The chondrogenic potential of MSCs was determined by staining for Alcian blue. $\mathbf{d}$ Cell surface markers of MSCs were assessed using flow cytometry. MSCs expressed CD29, CD44, and CD90, but not CD34, CD45 
experimental data. Statistically significant was represented as ${ }^{*} P<0.05,{ }^{* *} P<0.01$, and ${ }^{* * * *} P<0.001$.

\section{Results}

\section{Identification of BM-MSC characteristics}

Isolated cells were plastic-adherent in culture and displayed a typical fibroblast morphology as shown in Fig. 1a. mKate2 fluorescence was visible in the nucleus of the transduced cells demonstrated in Fig. 1b. Also, cultured cells were positive for oil red $\mathrm{O}$ staining, alizarin red staining, and Alcian blue staining (Fig. 1c). As shown in Fig. 1d, 95\% of these cells were positive for CD29, CD44, and CD90, but negative for CD34 and CD45 indicating that the cultured cells possessed the MSC characteristics [24].

\section{Characteristics of the T2DM and vasculopathy}

Blood glucose, IPGTTs, IPITTs, and serum insulin were measured to confirm the success of T2DM rat model. One week after STZ injection, blood glucose levels in the T2DM group increased to more than double that of normal rats $(p<0.001$, Fig. 2a). The results of IPGTTs revealed significant deterioration in glucose metabolism (Fig. 2b), and IPITTs showed a significant decrease in insulin sensitivity (Fig. 2c). However, there was no significant difference in serum insulin levels between the normal and T2DM rats (Fig. 2d). After 12 weeks of STZ injection, the bodyweight of T2DM rats was significantly lighter than normal rats $(p<0.01$, Fig. 2e). Moreover, T2DM rats required more water and feed than normal rats within $24 \mathrm{~h}(P<0.001$, Fig. $2 \mathrm{f})$. As shown in Fig. $2 \mathrm{~g}$, serum NO level in the T2DM group was lower than that in the normal group $(P<0.01)$. However, serum ET-1 levels in the T2DM group were higher than that in the normal group $(P<0.05)$. Besides, lipid accumulation was observed in the skin vessels, kidneys, and myocardium of T2DM rats (Fig. 2h).

\section{Both topical and systemic transplantation of BM-MSCs enhanced wound healing}

As shown in Fig. 3a, b, BM-MSCs treated wounds exhibited accelerated wound closure in topical and IV groups compared with wounds in the control group at all four time points $(p<0.05)$. In addition, the healing rate in the IV group was significantly higher than that in the topical group at days $3(P<0.01)$ and $10(P<0.05)$. Histologically, both topical and IV groups wound had complete epithelialization by day 14 (Fig. 4a). As shown in Fig. 4b, the length of the epithelial edges in both treatment groups was significantly longer than that in the control

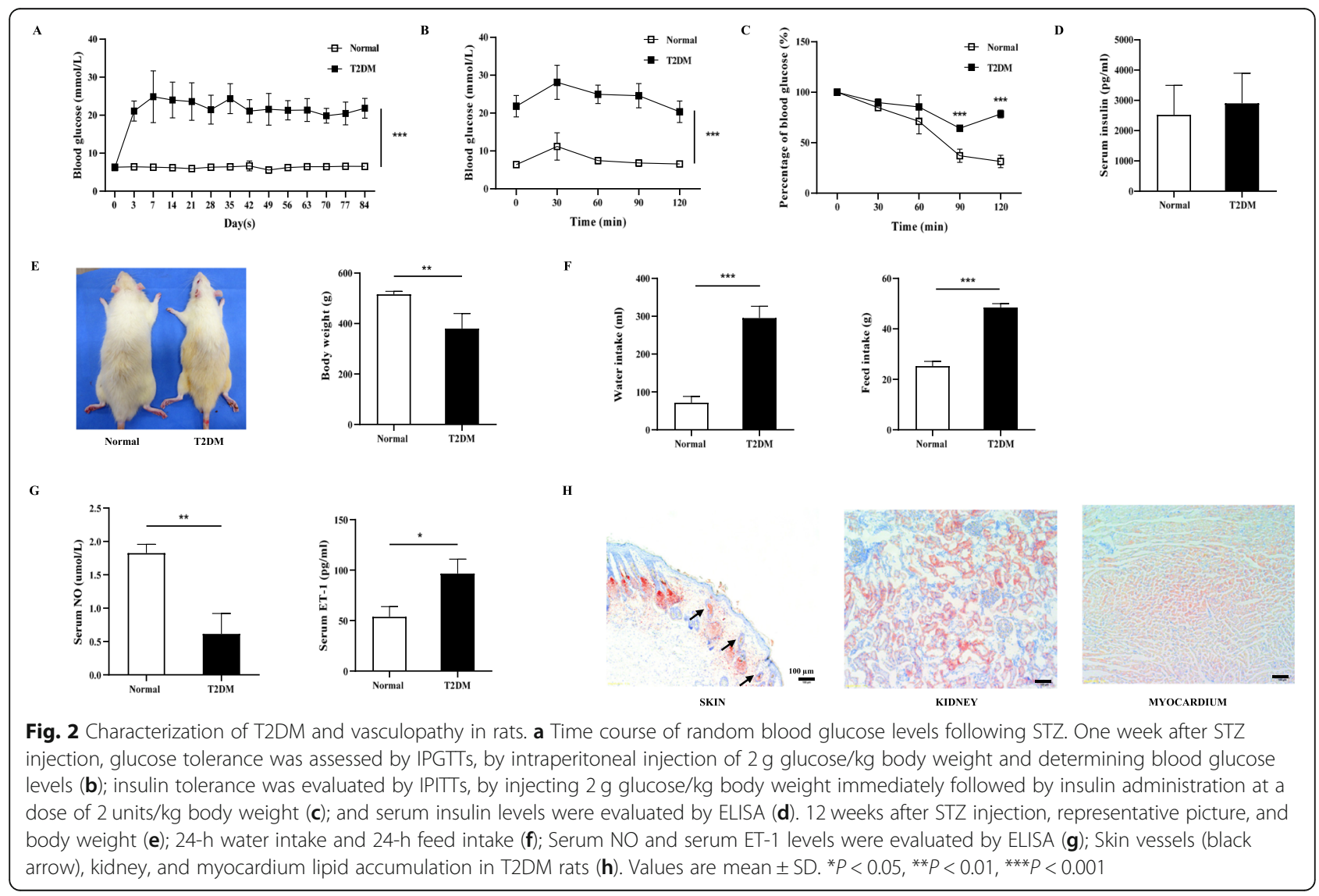




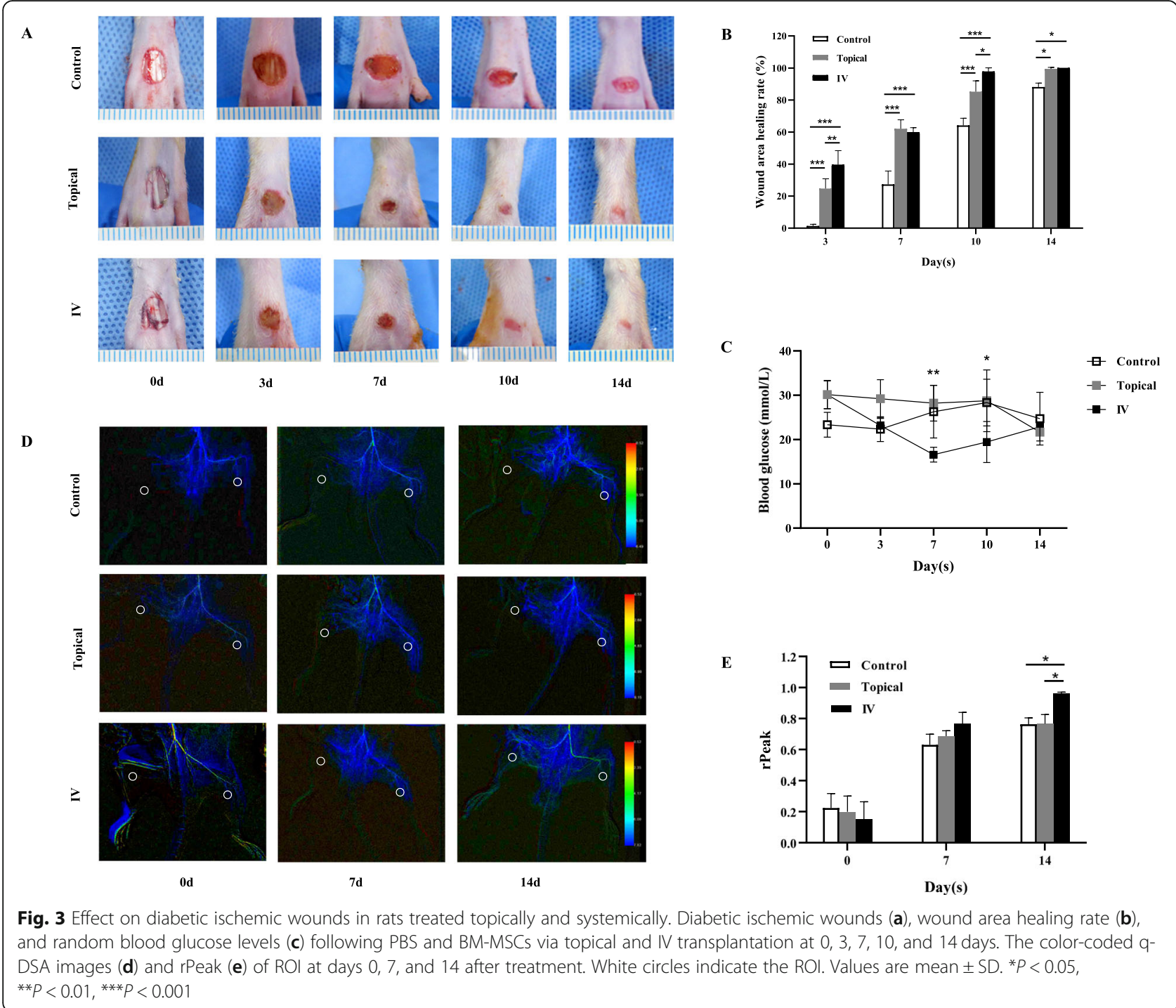

group at days 7,10 , and $14(P<0.001)$. In contrast, the length of the epithelial gap in the control group was longer longer than that of the topical and IV groups at days $3,7,10$, and 14 $(P<0.001)$. Meanwhile, the control group had a larger area of granulation tissue than in both topical and IV groups at day $14(P<0.001)$.

Histological analysis of Masson's trichrome staining and CD31 IHC staining sections revealed differences in the repair efficiency of the wounds among all three groups at different time points. As shown in Fig. 4a, granulation tissue in the control group was loose and accompanied by edema. By contrast, granulation tissue in topical and IV groups had tremendous collagen deposition with large wavy collagen fibers. The increase of collagen content in both topical and IV groups was greater than that of the control group at days $3(P<0.05), 7$ $(P<0.001), 10(P<0.001)$, and $14(P<0.001)$ (Fig. $4 \mathrm{~d})$. Furthermore, compared with the control group, IHC staining for CD31 revealed that more angiogenic formation in the wound of both topical and IV groups at day 3 to day 14 postwounding (Fig. $5 \mathrm{a}$ and $\mathrm{b}$ ).

\section{Systemic transplantation of BM-MSCs ameliorated hyperglycemia and improved blood perfusion of the ischemic limb}

As shown in Fig. 3c, a decline of the blood glucose level was present in the IV group at days 7 and 10 but still remained the initial level at day 14. Compared with the control and topical groups, the IV group had a better blood perfusion recovery of ischemic hindlimbs at day 14 ( $P<0.05$, Fig. 3d, e).

\section{The homing of mKate2-labeled BM-MSCs}

Representative fluorescence imaging of major organs are shown in Fig. 6c. In the IV group, the fluorescence signals was seen in the lungs, liver, pancreas, and kidney at 


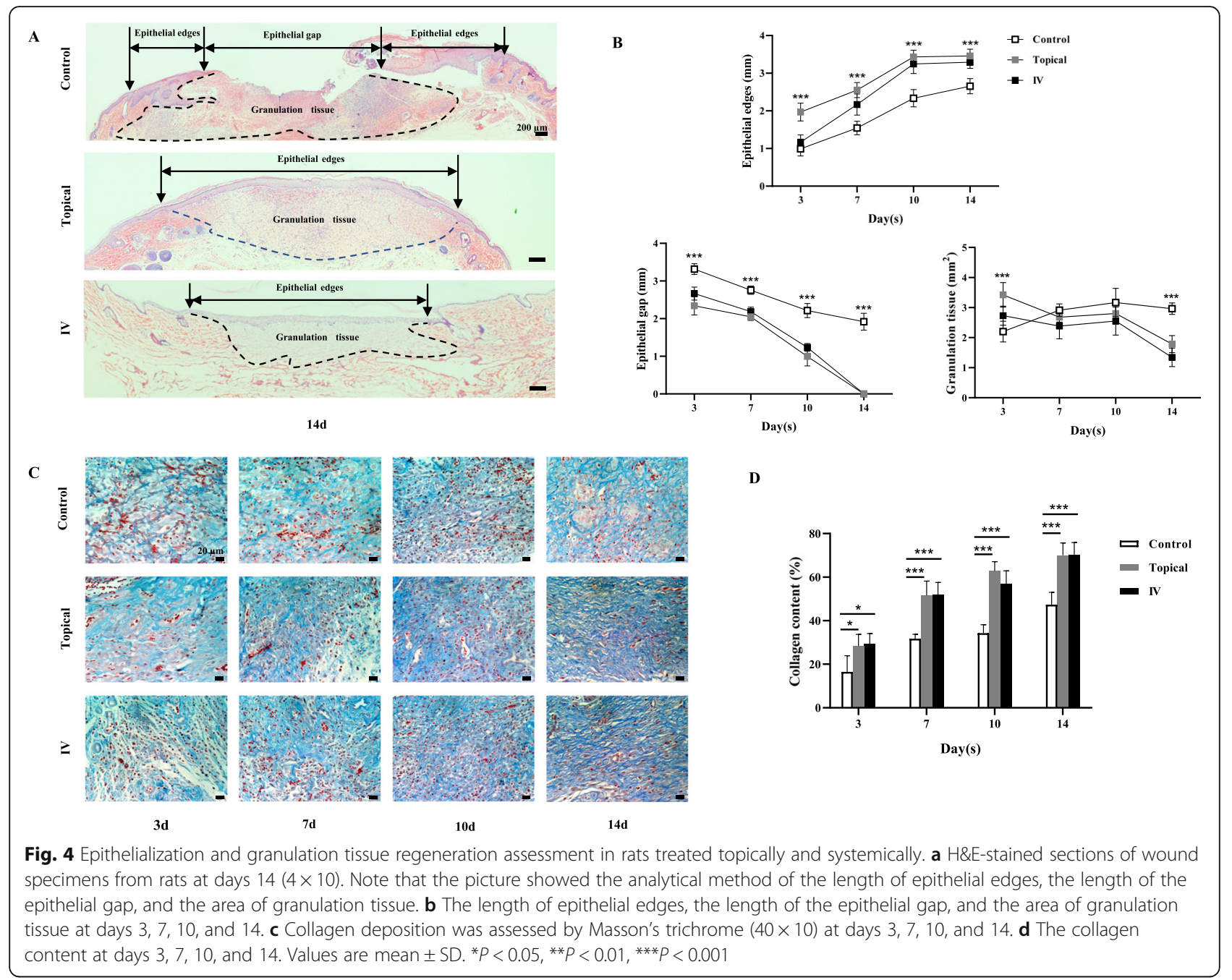

day 3. As time progressed, the fluorescence signals decreased and on day 14 signals were untraceable. However, no fluorescence signal could be detected in any of these organs in the topical group. As shown in Fig. 6d, mKate2 IHC analysis of the lungs, pancreas, and the wound was performed to further confirm the tracing allocation: red fluorescent protein was found in the lungs and pancreas in the IV group. Moreover, mKate2 staining was detected within the wound in both topical and IV group, with a larger stain area observed in the topical group compared to the IV group at days $3(P<0.001)$ and $7(P<0.01)$ (Fig. 6b, d).

\section{BM-MSCs transdifferentiated into endothelial cells and increased VEGF expression}

The transdifferentiation capacity of MSCs and expression of VEGF were assessed in the wound at day 14. As shown in Fig. 7a, mKate2-positive cells (red) were colocalized with CD31 (green) in wound site and right gastrocnemius tissue. Furthermore, VEGF content in the control group was lower than that of the topical and IV groups $(P<0.01$, Fig. $7 \mathrm{~b}, \mathrm{c})$. Western blot analysis of the wound tissues showed a reduction of PTEN, and an increase in the amount of p-AKT and VEGF protein expression in both topical and IV groups when compared with that of the control group (Fig. $7 d-g$ ).

\section{Discussion}

Many previous studies and clinical trials have shown that stem cells therapy and related biotechnology hold a great promise in regenerative medicine applications [2528]. BM-MSCs, adipose-derived mesenchymal stem cells (AD-MSCs), and stromal vascular fraction cells (SVFs) have the ability to promote wound healing and soft tissue defects when used alone [29-31] or in combination with platelet rich plasma (PRP) and fat graft [32-34]. Additionally, the most recent studies reported systemically administered MSCs improve respiratory activity in patients with coronavirus disease 2019 (COVID-19) [35-37]. However, we still lack treatment guidelines given the limited evidence from 
A

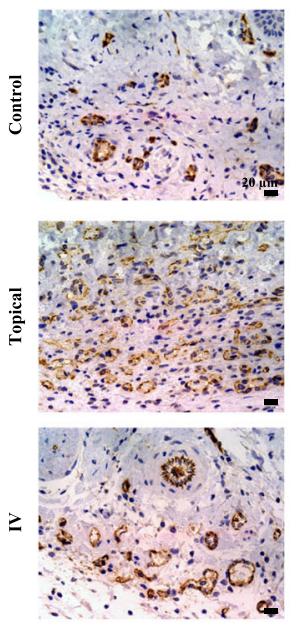

3d

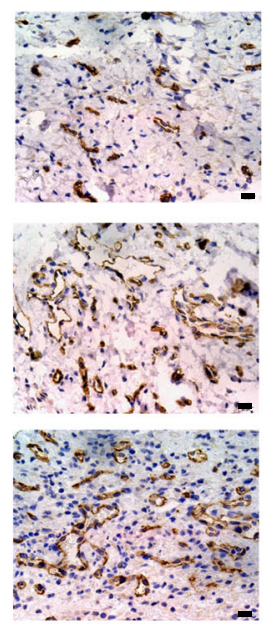

$7 d$

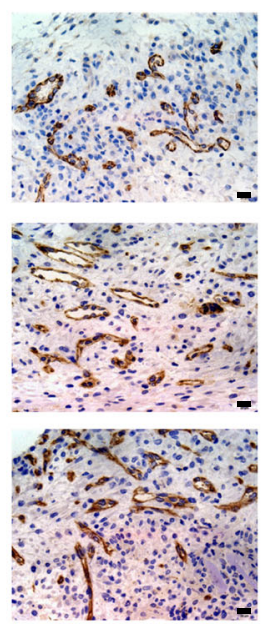

10d

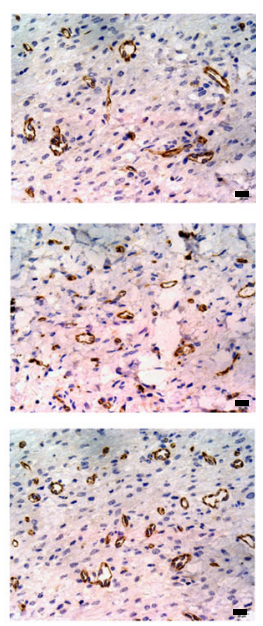

14d

B

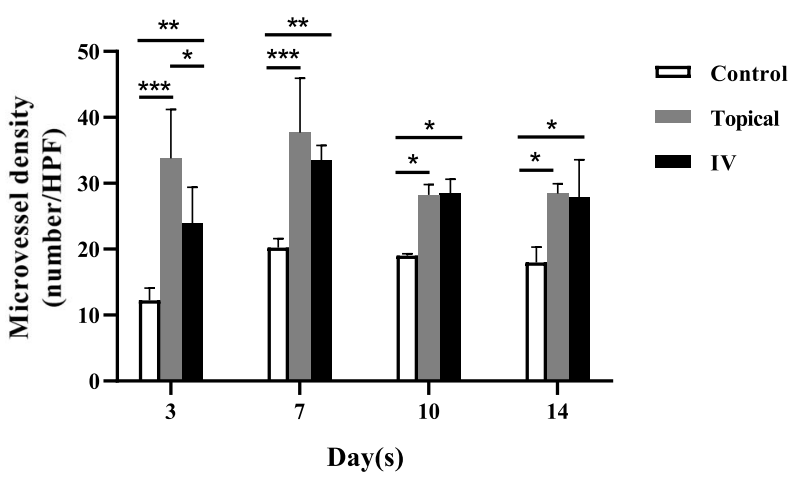

Fig. 5 Angiogenesis assessment in the wound. a IHC staining of CD31 $(40 \times 10)$ showed more microvessels on the wound in the topical and IV groups compared with the control group at days 3, 7, 10, and 14. b The microvessel density in the wound at days $3,7,10$, and 14 . Values are mean \pm SD. ${ }^{*} P<0.05,{ }^{* *} P<0.01,{ }^{* * *} P<0.001$

comparative research, which is becoming a crucial issue hindering the clinical application of MSCs. Regarding the route of administration, topical administration was the preferred mode of administration followed by systemic administration. Topical administration is considered to be an easy and effective route for MSCs delivery to treat chronic wounds [38, 39]. Systemic administration has also indicated that MSCs could accelerate cutaneous wound healing [6] . In addition, a recent study suggests that both topical and systemic administration has the potential to facilitate wound healing under physiological conditions [17]. In this study, we sought to compare topical and systemic transplantation of BM-MSCs to investigate the proper administration method for chronic wound therapy.

We established a novel animal model of DFUs that simulates clinical symptoms. This model exhibited hyperglycemia, metabolic disorder, atherosclerosis, hindlimb ischemia, and chronic wound. The findings of the present study suggested that both topically and systemically administered BM-MSC treatment result in accelerated wound closure, facilitated reepithelialization, improved granulation, and enhanced angiogenesis. Moreover, a transient decrease in blood glucose levels was observed after systemic transplantation of BM-MSCs, as reported previously [40]. Systemic administration also improved blood perfusion in the ischemic hindlimb steadily and continuously. We believe that these systemic effects originated from the route of administration.

As the most commonly used seed cells for tissue repair and regeneration, MSCs actively respond to biological signals associated with inflammation, necrosis, and tissue injury [41]. After systemic administration, BM-MSCs were observed at the skin wound sites $[42,43]$, and notably, they secreted bioactive factors to recruit host repair cells $[44,45]$. A plethora of chemokines, growth factors, and receptors were involved in the recruitment of MSCs [46-49]. In our study, compared with topical transplantation, BM-MSCs administered systemically was distributed in wounded tissues, such as pancreas and ischemic 
A
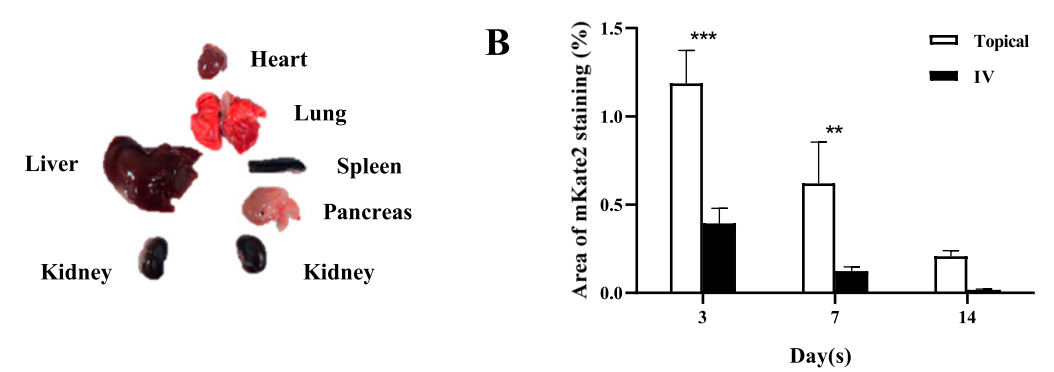

C
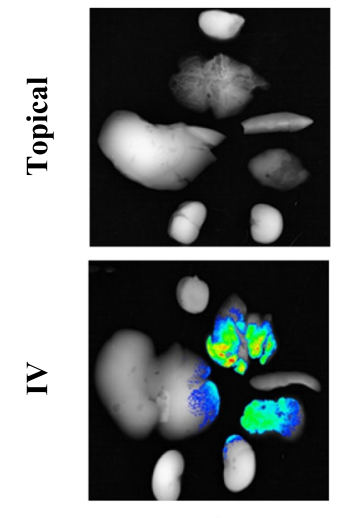

3d
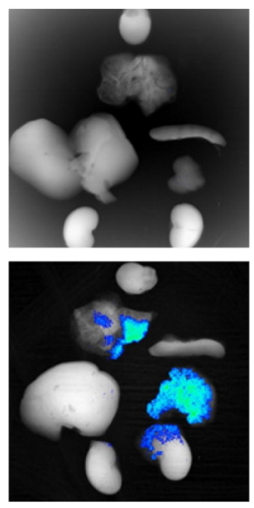

7d
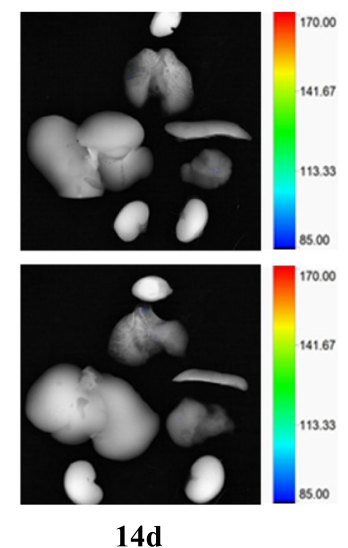

D
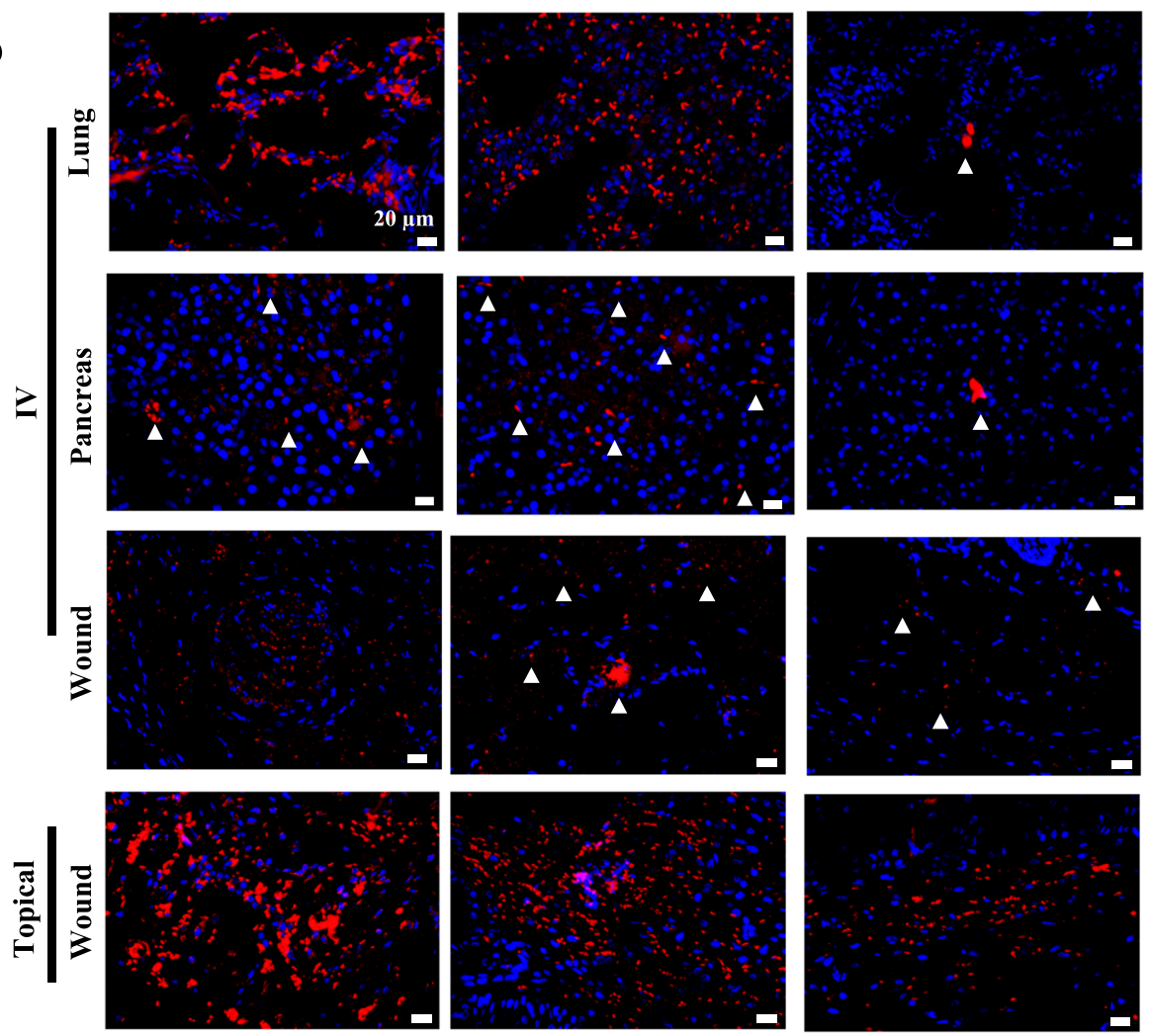

3d

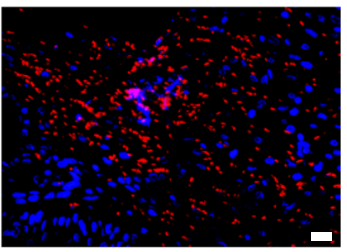

7d
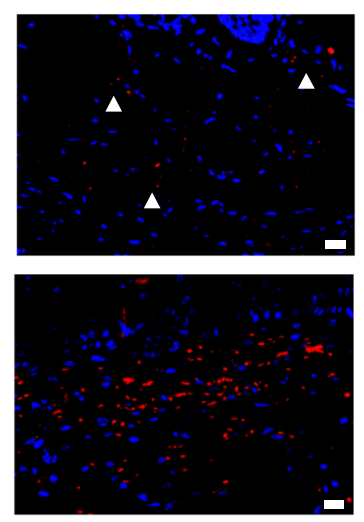

14d

Fig. 6 (See legend on next page.) 
(See figure on previous page.)

Fig. 6 In vivo tracking of mKate2-labeled BM-MSCs during wound repair under pathological conditions. a The appearance of major organs harvested from model rats. $\mathbf{b}$ The area of mKate2 staining on the wound at days 3, 7, and 14. c Fluorescence imaging of major organs harvested at days 3, 7, and 14 after transplantation of mKate2-labeled BM-MSCs. $\mathbf{d}$ mKate2-labeled BM-MSCs distribution analysis on lung, pancreas, and wound sections at days 3,7 , and 14 posttransplantation via IV and topical, respectively (RFP IHC staining, $40 \times 10$ ). Values are mean \pm SD. ${ }^{*} P<0.05$, ${ }^{* *} P<0.01,{ }^{* * *} P<0.001$

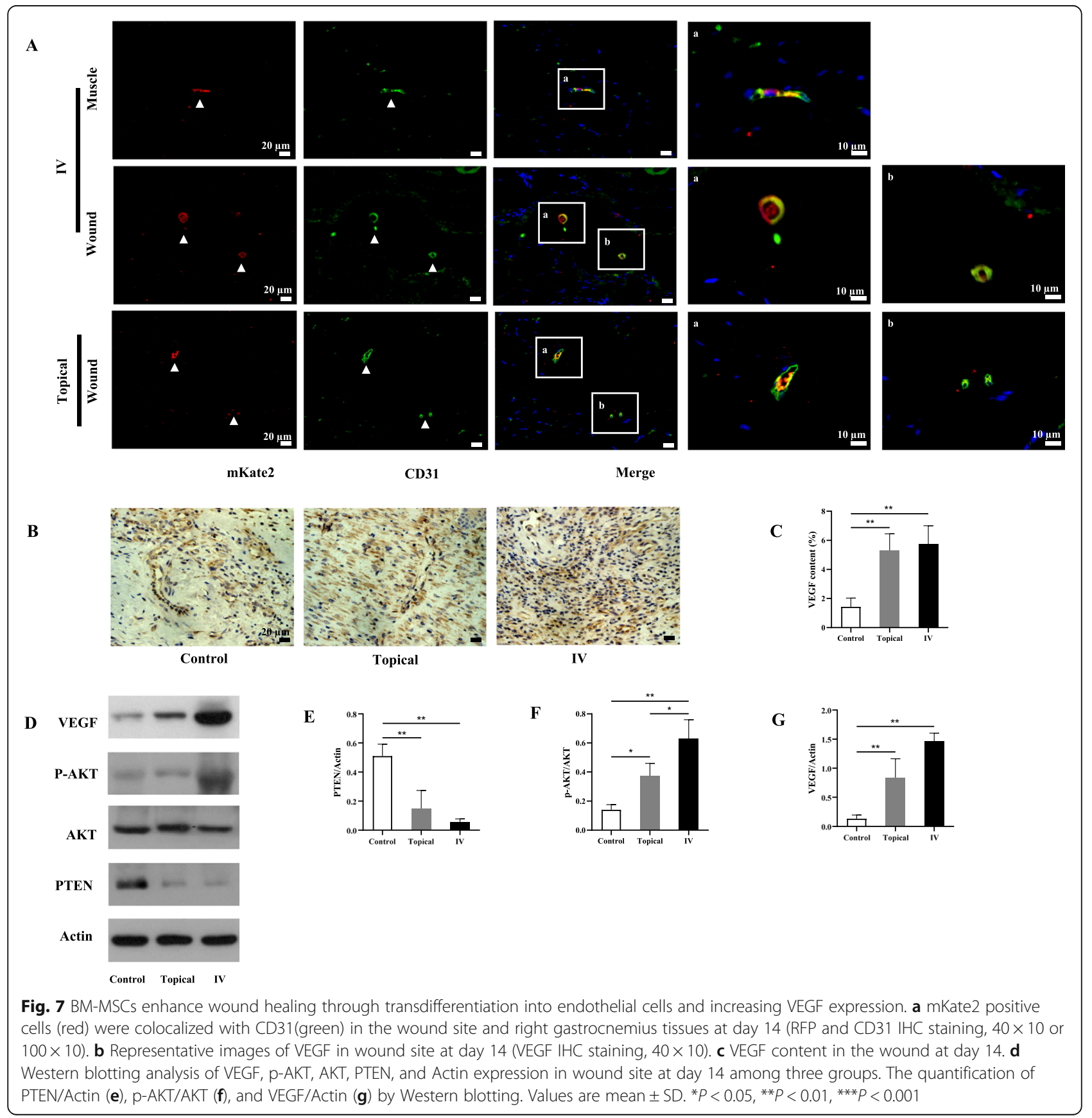


muscle. Our study also revealed that BM-MSCs can transdifferentiate into endothelial cells in the ischemic muscle. Therefore, that might be the reason for ameliorating hyperglycemia and improving blood perfusion of ischemic hindlimb after systemic application.

The mechanisms of MSC-based wound therapy have not been fully delineated, yet two mechanisms are generally postulated: (1) direct differentiation into skin cells [50] and (2) the secretion of trophic factors [51]. Considering the poor engraftment of MSCs at chronic wounds, it is widely believed that the therapeutic effects depend predominantly on their paracrine actions by which MSCs secrete a multitude of soluble factors, such as growth factors, immune factors, chemokines, and exosomes, to enhance the survival, recruitment, and function of wound repair cells [25]. The latest research suggests that systemic transplantation of MSCs have the potential to promote wound repair possibly by paracrine effect; however, topical transplantation of MSCs enhanced wound repair due to redistribution in the wound bed [17]. In this article, we showed that both the topical and systemic transplantation of BM-MSCs have the ability to migrate into wound sites and transdifferentiate into endothelial cells. Furthermore, compared with systemic administration, more BM-MSCs were found in the wound after topical administration. However, topical and systemic transplantation of BM-MSCs had no significant differences in wound healing. One explanation may be that systemic delivery of MSCs exerts a stronger paracrine effect than topical administration.

It is believed that angiogenesis is an important part of tissue repair [52]. As a potent growth and angiogenic cytokine, VEGF is involved in the formation and maturation of blood vessels [53]. The results from this study showed that VEGF expression increased in wounds after BM-MSC transplantation. It is established that the AKT signaling pathway is an important pathway regulating angiogenesis [54]. p-AKT activates VEGF signaling pathway, which leads to cell proliferation and angiogenesis [55]. In contrast, PTEN inhibits AKT signaling pathway through dephosphorylation [56]. In the present study, the wound following BM-MSC treatment exhibited the level of p-AKT increased while the level of PTEN decreased. Our findings demonstrate that BM-MSCs improve wound healing through regulating angiogenesis by targeting the PTEN/AKT pathway.

\section{Conclusion}

In summary, we demonstrated that both topical and systemic transplantation of BM-MSCs accelerated wound healing remarkably under pathological conditions. This was evidenced in a diabetic ischemic wound model. At the same time, systemic administration has the potential to ameliorate hyperglycemia and repair the damaged tissue. The mechanism involved transdifferentiation into endothelial cells, stimulating VEGF secretion and activating the AKT signaling pathways. The obtained data provide new evidence for the potential application of MSC transplantation in chronic wound therapy.

\section{Abbreviations}

MSCs: Mesenchymal stem cells; T2DM: Type 2 diabetes mellitus; BMMSCs: Bone marrow-derived mesenchymal stem cells; IV: Intravenous; IHC: Immunohistochemistry; VEGF: Vascular endothelial growth factor; DFUs: Diabetic foot ulcers; PAD: Peripheral arterial disease;

STZ: Streptozotocin; DMEM: Dulbecco's modified Eagle's medium; FBS: Fetal bovine serum; pen/strep: Penicillin-streptomycin; CD29: Cluster of differentiation 29; CD44: Cluster of differentiation 44; CD90: Cluster of differentiation 90; CD34: Cluster of differentiation 34; CD45: Cluster of differentiation 45; IPGTTs: Intraperitoneal glucose tolerance tests; IPIT Ts: Intraperitoneal insulin tolerance tests; ELISA: Enzyme-linked immunosorbent assay; NO: Nitric oxide; ET-1: Serum endothelin-1; PBS: Phosphate-buffered saline; q-DSA: Quantitative digital subtraction angiography; ROI: Region of interest; H\&E: Hematoxylin and eosin; HPF: High power field; CD31: Cluster of differentiation 31; DAPI: 4',6-Diamidino-2phenylindole; PTEN: Phosphatase and tensin homolog; $\mathrm{p}$ -

AKT: Phosphorylation of AKT; AD-MSCs: Adipose-derived mesenchymal stem cells; SVFs: Stromal vascular fraction cells; PRP: Platelet rich plasma

\section{Acknowledgements}

Not applicable

\section{Authors' contributions}

JXY: Conception and design, provision of study material, collection of data, data analysis, manuscript writing, and final approval of the manuscript. JاL: Conception and design, provision of study material, collection of data, data analysis, manuscript writing, and final approval of the manuscript. YXC: Collection of data, data analysis, and final approval of the manuscript. ME: Collection of data, data analysis, and final approval of the manuscript. XL: Collection of data, data analysis, and final approval of the manuscript. XJC: Collection of data, data analysis, and final approval of the manuscript. KCL: Collection of data, data analysis, and final approval of the manuscript. GHX: Concepcion and design, data analysis, and final approval of the manuscript. HWL: Conception and design, financial support, data analysis, manuscript writing, and final approval of the manuscript.

\section{Funding}

This study was supported by the National Nature and Science Foundation of China (81871563 and 81372065), the Fundamental Research Funds for the Central Universities (21619350), the foster research fund of The First Affiliated Hospital of Jinan University (2017306), the Fundamental Research Funds for the Central Universities (21619357), and Guangdong Medical Science and Technology Research Foundation (A2020270).

Availability of data and materials

All data generated or analyzed during this study are included in this published article.

\section{Declarations}

Ethics approval and consent to participate

The Experimental Animal Ethics Committee of Jinan University approved the animal experiments for this study.

Consent for publication

Not applicable

\section{Competing interests}

The authors declare that they have no competing interests.

\section{Author details}

${ }^{1}$ Department of Plastic Surgery, The First Affiliated Hospital of Jinan University, Guangzhou 510630, People's Republic of China. ${ }^{2}$ Innovative Technology Research Institute of Plastic Surgery, Guangzhou 510630, 
People's Republic of China. ${ }^{3}$ Key Laboratory of Regenerative Medicine, Ministry of Education, Guangzhou 510632, People's Republic of China. ${ }^{4}$ Department of Cell Biology \& Institute of Biomedicine, College of Life Science and Technology, Jinan University, Guangzhou 510632, People's Republic of China. ${ }^{5}$ Guangdong Province Key Laboratory of Bioengineering Medicine, Guangzhou 510632, People's Republic of China. ${ }^{6}$ Guangdong Provincial Biotechnology Drug \& Engineering Technology Research Center, Guangzhou 510632, People's Republic of China. ${ }^{7}$ National Engineering Research Center of Genetic Medicine, Guangzhou 510632, People's Republic of China. ${ }^{8}$ Department of Interventional Radiology and Vascular Surgery, The First Affiliated Hospital of Jinan University, Guangzhou 510630, People's Republic of China.

\section{Received: 30 January 2021 Accepted: 15 March 2021} Published online: 31 March 2021

\section{References}

1. Nyenwe EA, Jerkins TW, Umpierrez GE, Kitabchi AE. Management of type 2 diabetes: evolving strategies for the treatment of patients with type 2 diabetes. Metabolism. 2011;60(1):1-23. https://doi.org/10.1016/j.metabol.201 0.09.010.

2. Yazdanpanah L, Nasiri M, Adarvishi S. Literature review on the management of diabetic foot ulcer. World J Diabetes. 2015;6(1):37-53. https://doi.org/1 0.4239/wjd.v6.i1.37.

3. Shiekh PA, Singh A, Kumar A. Exosome laden oxygen releasing antioxidant and antibacterial cryogel wound dressing OxOBand alleviate diabetic and infectious wound healing. Biomaterials. 2020;249:120020.

4. Lewis JE, Morris K, Powell T, et al. Combining diabetic foot and retinopathy screening: a step in the right direction?-a feasibility study. SAGE Open Med. 2020;8:2050312120946244.

5. Lim JZ, Ng NS, Thomas C. Prevention and treatment of diabetic foot ulcers. J R Soc Med. 2017;110(3):104-9. https://doi.org/10.1177/0141076816688346.

6. Shi R, Jin Y, Cao C, Han S, Shao X, Meng L, Cheng J, Zhang M, Zheng J, Xu J, Li M. Localization of human adipose-derived stem cells and their effect in repair of diabetic foot ulcers in rats. Stem Cell Res Ther. 2016;7(1):155. https://doi.org/10.1186/s13287-016-0412-2.

7. Kato J, Kamiya H, Himeno T, Shibata T, Kondo M, Okawa T, Fujiya A, Fukami A, Uenishi E, Seino Y, Tsunekawa S, Hamada Y, Naruse K, Oiso Y, Nakamura J. Mesenchymal stem cells ameliorate impaired wound healing through enhancing keratinocyte functions in diabetic foot ulcerations on the plantar skin of rats. J Diabetes Complications. 2014;28(5):588-95. https://doi.org/10.1 016/j.jdiacomp.2014.05.003.

8. Si Y-L, Zhao Y-L, Hao H-J, Fu XB, Han WD. MSCs: biological characteristics, clinical applications and their outstanding concerns. Ageing Res Rev. 2011; 10(1):93-103. https://doi.org/10.1016/j.arr.2010.08.005.

9. Ankrum J, Karp JM. Mesenchymal stem cell therapy: two steps forward, one step back. Trends Mol Med. 2010;16(5):203-9. https://doi.org/10.1016/j. molmed.2010.02.005.

10. Gupta PK, Chullikana A, Parakh R, Desai S, Das A, Gottipamula S, Krishnamurthy S, Anthony N, Pherwani A, Majumdar AS. A double blind randomized placebo controlled phase I/II study assessing the safety and efficacy of allogeneic bone marrow derived mesenchymal stem cell in critical limb ischemia. J Transl Med. 2013;11(1):1-11. https://doi.org/10.11 86/1479-5876-11-143.

11. Vojtassák J, Danisovic L, Kubes M, et al. Autologous biograft and mesenchymal stem cells in treatment of the diabetic foot. Neuro Endocrinol Lett. 2006;27(Suppl 2):134.

12. Sarasúa JG, López SP, Viejo MA, et al. Treatment of pressure ulcers with autologous bone marrow nuclear cells in patients with spinal cord injury. J Am Paraplegia Soc. 2011;34(3):301-7.

13. Karp JM, Teo GSL. Mesenchymal stem cell homing: the devil is in the details. Cell Stem Cell. 2009;4(3):206-16. https://doi.org/10.1016/j.stem.2009. 02.001 .

14. Li Z, Hu X, Mao J, Liu X, Zhang L, Liu J, Li D, Shan H. Optimization of mesenchymal stem cells (MSCs) delivery dose and route in mice with acute liver injury by bioluminescence imaging. Mol Imaging Biol. 2015;17(2):18594. https://doi.org/10.1007/s11307-014-0792-6.

15. Spriet M, Hunt GB, Walker NJ, Borjesson DL. Scintigraphic tracking of mesenchymal stem cells after portal, systemic intravenous and splenic administration in healthy beagle dogs. Vet Radiol Ultrasound. 2015:56(3): 327-34. https://doi.org/10.1111/vru.12243.
16. Jun Soo B, Byung Kook K, Jae Kyun K, et al. Engraftment of human mesenchymal stem cells in a rat photothrombotic cerebral infarction mode : comparison of intra-arterial and intravenous infusion using MRI and histological analysis. J Korean Neurosurg Soc. 2013;54(6):467-76.

17. Kallmeyer K, André-Lévigne D, Baquié M, Krause KH, Pepper MS, PittetCuénod B, Modarressi A. Fate of systemically and locally administered adipose-derived mesenchymal stromal cells and their effect on wound healing. Stem Cells Transl Med. 2020;9(1):131-44. https://doi.org/10.1002/ sctm.19-0091.

18. Bieback K, Kern S, Klüter $H$, et al. Critical parameters for the isolation of mesenchymal stem cells from umbilical cord blood. Stem Cells. 2010;22(4): 625-34.

19. Reed MJ, Meszaros K, Entes LJ, Claypool MD, Pinkett JG, Gadbois TM, Reaven GM. A new rat model of type 2 diabetes: the fat-fed, streptozotocin-treated rat. Metab Clin Exp. 2000;49(11):1390-4. https://doi.org/10.1053/meta.2000.1 7721

20. Marsh SA, Dell'Italia LJ, Chatham JC. Interaction of diet and diabetes on cardiovascular function in rats. Am J Physiol Heart Circ Physiol. 2009;296(2): H282-92. https://doi.org/10.1152/ajpheart.00421.2008.

21. Wu Y, Chen L, Scott PG, Tredget EE. Mesenchymal stem cells enhance wound healing through differentiation and angiogenesis. Stem Cells. 2007; 25(10):2648-59. https://doi.org/10.1634/stemcells.2007-0226.

22. Jian-Xin, Yan, Xuan et al. Effects of Carbon Arc Lamp Irradiation on Wound Healing in a Rat Cutaneous Full-Thickness Wound Model. Photobiomodul Photomed Laser Surg. 2019;37(1):17-24.

23. Evans M, Baddour HM Jr, Magliocca KR, et al. Prognostic implications of peritumoral vasculature in head and neck cancer. Cancer Med. 2019;8(1): 147-54. https://doi.org/10.1002/cam4.1910.

24. Dominici M, Blanc KL, Mueller I, et al. Minimal criteria for defining multipotent mesenchymal stromal cells. The International Society for Cellular Therapy position statement. Cytotherapy. 2006;8(4):315.

25. Huang YZ, Gou M, Da LC, et al. Mesenchymal stem cells for chronic wound healing: current status of preclinical and clinical studies. Tissue Eng Part B Rev. 2020;26(6):555-70. https://doi.org/10.1089/ten.teb.2019.0351.

26. Pietro G, Ashutosh K, Donato $C$, et al. Fat graft enhanced with adiposederived stem cells in aesthetic breast augmentation: clinical, histological, and instrumental evaluation. Aesthet Surg J. 2019;9:9.

27. Gentile P, Sterodimas A, Calabrese C, de Angelis B, Trivisonno A, Pizzicannella J, Dionisi L, de Fazio D, Garcovich S. Regenerative application of stromal vascular fraction cells enhanced fat graft maintenance: clinical assessment in face rejuvenation. Expert Opin Biol Ther. 2020;20(12):1503-13. https://doi.org/10.1080/14712598.2020.1815703.

28. Gentile P, Casella D, Palma E, Calabrese C. Engineered fat graft enhanced with adipose-derived stromal vascular fraction cells for regenerative medicine: clinical, histological and instrumental evaluation in breast reconstruction. J Clin Med. 2019;8(4):504. https://doi.org/10.3390/jcm804 0504.

29. Gentile P, Sterodimas A, Pizzicannella J, Dionisi L, de Fazio D, Calabrese C, Garcovich S. Systematic review: allogenic use of stromal vascular fraction (SVF) and decellularized extracellular matrices (ECM) as advanced therapy medicinal products (ATMP) in tissue regeneration. Int J Mol Sci. 2020;21(14): 4982. https://doi.org/10.3390/ijms21144982.

30. Gentile P, Garcovich S. Concise review: adipose-derived stem cells (ASCs) and adipocyte-secreted exosomal microRNA (A-SE-miR) modulate cancer growth and promote wound repair. J Clin Med. 2019;8(6):855.

31. Pietro G, Scioli, et al. Comparing different nanofat procedures on scars: role of the stromal vascular fraction and its clinical implications. Regen Med. 2017:12:9392018.

32. Maria G, Scioli, et al. Combined treatment with platelet-rich plasma and insulin favours chondrogenic and osteogenic differentiation of human adipose-derived stem cells in three-dimensional collagen scaffolds. J Tissue Eng Regen Med. 2017;11(8):2398-410.

33. Gentile P, Scioli MG, Bielli A, et al. Concise review: the use of adiposederived stromal vascular fraction cells and platelet rich plasma in regenerative plastic surgery. Stem Cells. 2017;35(1):117-34.

34. Gentile P, De Angelis B, Pasin M, et al. Adipose-derived stromal vascular fraction cells and platelet-rich plasma: basic and clinical evaluation for cellbased therapies in patients with scars on the face. J Craniofac Surg. 2014; 25(1):267-72. https://doi.org/10.1097/01.scs.0000436746.21031.ba.

35. Gentile P, Sterodimas A, Pizzicannella J, Calabrese C, Garcovich S. Research progress on mesenchymal stem cells (MSCs), adipose-derived mesenchymal 
stem cells (AD-MSCS), drugs, and vaccines in inhibiting COVID-19 disease. Aging Dis. 2020;11(5):1191-201. https://doi.org/10.14336/AD.2020.0711.

36. Gentile P, Sterodimas A. Adipose stem cells (ASCs) and stromal vascular fraction (SVF) as a potential therapy in combating (COVID-19)-disease. Aging Dis. 2020;11(3):465-9. https://doi.org/10.14336/AD.2020.0422.

37. Gentile P, Sterodimas A. Adipose-derived stromal stem cells (ASCs) as a new regenerative immediate therapy combating coronavirus (COVID-19)-induced pneumonia. Expert Opin Biol Ther. 2020;20(7):711-6. https://doi.org/10.1 080/14712598.2020.1761322.

38. Wu Y, Chen L, Scott PG et al. Mesenchymal stem cells enhance wound healing through differentiation and angiogenesis. Stem Cells. 2007;25(10): 2648-59.

39. Suzdaltseva Y, Zhidkih S, Kiselev SL, Stupin V. Locally delivered umbilical cord mesenchymal stromal cells reduce chronic inflammation in long-term nonhealing wounds: a randomized study. Stem Cells Int. 2020;2020(4):1-11. https://doi.org/10.1155/2020/5308609.

40. Si Y, Zhao Y, Hao H, Liu J, Guo Y, Mu Y, Shen J, Cheng Y, Fu X, Han W. Infusion of mesenchymal stem cells ameliorates hyperglycemia in type 2 diabetic rats. Diabetes. 2012;61(6):1616-25. https://doi.org/10.2337/ db11-1141.

41. Prockop DJ, Oh JY. Mesenchymal stem/stromal cells (MSCs): role as guardians of inflammation. Mol Ther. 2012;20(1):14-20. https://doi.org/10.1 038/mt.2011.211.

42. Oh EJ, Lee HW, Kalimuthu S, Kim TJ, Kim HM, Baek SH, Zhu L, Oh JM, Son SH, Chung HY, Ahn BC. In vivo migration of mesenchymal stem cells to burn injury sites and their therapeutic effects in a living mouse model. J Control Release. 2018;279:79-88. https://doi.org/10.1016/j.jconrel.2018.04.020.

43. Chen G, Tian F, Li C, Zhang Y, Weng Z, Zhang Y, Peng R, Wang Q. In vivo real-time visualization of mesenchymal stem cells tropism for cutaneous regeneration using NIR-II fluorescence imaging. Biomaterials. 2015;53:26573. https://doi.org/10.1016/j.biomaterials.2015.02.090.

44. Jiang D, Qi Y, Walker NG, Sindrilaru A, Hainzl A, Wlaschek M, MacNeil S, Scharffetter-Kochanek K. The effect of adipose tissue derived MSCs delivered by a chemically defined carrier on full-thickness cutaneous wound healing. Biomaterials. 2013;34(10):2501-15. https://doi.org/10.1016/j.biomaterials.2 012.12.014.

45. Formigli L, Paternostro F, Tani A, Mirabella C, Quattrini Li A, Nosi D, D'Asta F, Saccardi R, Mazzanti B, Lo Russo G, Zecchi-Orlandini S. MSCs seeded on bioengineered scaffolds improve skin wound healing in rats. Wound Repair Regen. 2015;23(1):115-23. https://doi.org/10.1111/wrr.12251.

46. Hocking AM. The role of chemokines in mesenchymal stem cell homing to wounds. Adv Wound Care. 2015;4(11):623-30. https://doi.org/10.1089/ wound.2014.0579.

47. Marquez-Curtis LA, Janowska-Wieczorek A. Enhancing the migration ability of mesenchymal stromal cells by targeting the SDF-1/CXCR4 axis. Biomed Res Int. 2013;2013:1-15. https://doi.org/10.1155/2013/561098.

48. Son BR, Marquez-Curtis LA, Kucia M, Wysoczynski M, Turner AR, Ratajczak J, Ratajczak MZ, Janowska-Wieczorek A. Migration of bone marrow and cord blood mesenchymal stem cells in vitro is regulated by stromal-derived factor-1-CXCR4 and hepatocyte growth factor-c-met axes and involves matrix metalloproteinases. Stem Cells. 2006;24(5):1254-64. https://doi.org/1 0.1634/stemcells.2005-0271.

49. Yu Y, Wu R-X, Gao L-N, Xia Y, Tang HN, Chen FM. Stromal cell-derived factor-1-directed bone marrow mesenchymal stem cell migration in response to inflammatory and/or hypoxic stimuli. Cell Adhes Migr. 2016; 10(4):1-18. https://doi.org/10.1080/19336918.2016.1139287.

50. Sasaki M, Abe R, Fujita Y, Ando S, Inokuma D, Shimizu H. Mesenchymal stem cells are recruited into wounded skin and contribute to wound repair by transdifferentiation into multiple skin cell type. J Immunol. 2008;180(4): 2581-7. https://doi.org/10.4049/jimmunol.180.4.2581.

51. Liang $X$, Zhang $Y$, Ding $Y$, et al. Paracrine mechanisms of mesenchymal stem cell-based therapy: current status and perspectives. Cell Transplant. 2013;23(9):1045.

52. Bi H, Li H, Zhang $\mathrm{C}$ et al. Stromal vascular fraction promotes migration of fibroblasts and angiogenesis through regulation of extracellular matrix in the skin wound healing process. Stem Cell Res Ther. 2019;10(1):302.

53. Yuan X, Han L, Fu P et al. Cinnamaldehyde accelerates wound healing by promoting angiogenesis via up-regulation of PI3K and MAPK signaling pathways. Lab Invest. 2018;98(6):783-98.
54. Somanath PR, Razorenova OV, Chen J, Byzova TV. Akt1 in endothelial cell and angiogenesis. Cell Cycle. 2006;5(5):512-8. https://doi.org/10.4161/cc.5. 5.2538 .

55. Fulton D, Gratton JP, Mccabe TJ, et al. Regulation of endothelium-derived nitric oxide production by the protein kinase Akt. Nature. 1999;399(6736): 597-601.

56. Ding J, Wang $X$, Chen B, et al. Exosomes derived from human bone marrow mesenchymal stem cells stimulated by deferoxamine accelerate cutaneous wound healing by promoting angiogenesis. Biomed Res Int. 2019;2019(7):1-12.

\section{Publisher's Note}

Springer Nature remains neutral with regard to jurisdictional claims in published maps and institutional affiliations.
Ready to submit your research? Choose BMC and benefit from:

- fast, convenient online submission

- thorough peer review by experienced researchers in your field

- rapid publication on acceptance

- support for research data, including large and complex data types

- gold Open Access which fosters wider collaboration and increased citations

- maximum visibility for your research: over $100 \mathrm{M}$ website views per year

At BMC, research is always in progress.

Learn more biomedcentral.com/submissions 